\title{
On some problems regarding growth analysis of composite entire and meromorphic function
}

\author{
SANJIB KUMAR DATTA AND TANMAY BISWAS
}

\author{
Date of Receiving $\quad$ : $\quad 20.11 .2016$ \\ Date of Revision : : 08.04 .2017 \\ Date of Acceptance : $\quad 09.04 .2017$
}

\begin{abstract}
In the paper we establish some new results depending on the comparative growth properties of composite entire or meromorphic functions using relative $L^{*}$-order, relative $L^{*}$ - type and relative $L^{*}$-weak type.
\end{abstract}

\section{Introduction, Definitions and Notations.}

Let $f$ be an entire function defined in the open complex plane $\mathbb{C}$. The maximum modulus function $M_{f}(r)$ corresponding to $f$ is defined on $|z|=r$ as follows:

$$
M_{f}(r)=\underset{|z|=r}{\max }|f(z)| .
$$

When $f$ is meromorphic, $M_{f}(r)$ cannot be defined as $f$ is not analytic throughout the complex plane. In this situation, one may introduce another function $T_{f}(r)$ known as Nevanlinna's characteristic function of $f$, playing the same role as maximum modulus function in the following manner:

$$
T_{f}(r)=N_{f}(r)+m_{f}(r),
$$

where

$$
N_{f}(r)=\int_{0}^{r} \frac{n_{f}(t)-n_{f}(0)}{t} d t+n_{f}(0) \log r
$$

2010 Mathematics Subject Classification. 30D35, 30D30, 30D20 .

Key words and phrases. Entire function, meromorphic function, composition, growth, relative $L^{*}$-order, relative $L^{*}$-type, relative $L^{*}$-weak type, slowly changing function.

The authors are thankful to referee for his/her valuable suggestions towards the improvement of the paper.

Communicated by: Harina P. Waghamore 